\title{
Ocean Surface Observations Using the TanDEM-X Satellite Formation
}

\author{
Steffen Suchandt and Hartmut Runge
}

\begin{abstract}
The TanDEM-X SAR satellite formation permits improved ocean surface observations by means of bistatic alongtrack interferometry (ATI) when compared to single-satellite systems. The flexible imaging geometry of its two cooperating SAR sensors forms an interferometer that can achieve very high sensitivity to motions of objects on ground. This way, radar imaging of surface currents with unprecedented accuracy, high spatial resolution and wide coverage at the same time becomes possible. We demonstrate the capabilities of the sensor in the contexts of tidal current mapping, measurement of thermohaline and wind-driven ocean currents as well as detection of areas with surface films. We have developed a dedicated postprocessing system for TanDEM-X image products that allows extracting surface current information from the data. By this paper, we address bistatic data acquisition and processing aspects for sea surface imaging with TanDEM-X like interferometric baseline geometry, temporal decorrelation, and phase calibration. We present a variety of examples of data evaluation that clearly demonstrate the application potential of the methodology.
\end{abstract}

Index Terms-Ocean surface currents, SAR along-track interferometry (ATI), TanDEM-X.

\section{INTRODUCTION}

$\mathbf{T}$ HE LARGE-AREA observation of oceans by means of spaceborne SAR sensors can provide valuable information in different application contexts such as climate research, energy harvesting, and environmental monitoring. Motion of the sea surface is associated with thermohaline and wind-driven circulations, tidal currents, and waves. SAR along-track interferometry (ATI) was shown to be a suitable technique to image these phenomena. This method utilizes at least two receive apertures along the sensor's flight track to repeatedly image the sea surface with a time lag $\Delta t=B_{A T I, \text { eff }} / v_{s}$, where $B_{A T I, \text { eff }}$ and $v_{s}$ are the effective ATI baseline, i.e., the alongtrack separation of the receive aperture's phase centers and the sensor velocity [1]. The phase difference $\phi_{A T I}$ between the signals of the spatially coregistered images is a measure of the line-of-sight (LOS) component of the ground motion $v_{L O S}$. The suitability of the technique was repeatedly demonstrated with single platform sensors like SRTM/X-SAR and TerraSAR-X [2], [3]. One drawback of those ATI systems is

Manuscript received March 01, 2015; revised May 28, 2015; accepted June 03, 2015. Date of publication August 18, 2015; date of current version January 18, 2016. This work was supported by the networking fund of the Helmholtz Association in the frame of the Helmholtz Alliance "Remote Sensing and Earth System Dynamics."

The authors are with the Remote Sensing Technology Institute, German Aerospace Center DLR, D-82234 Wessling, Germany (e-mail: steffen. suchandt@dlr.de; hartmut.runge@dlr.de).

Color versions of one or more of the figures in this paper are available online at http://ieeexplore.ieee.org.

Digital Object Identifier 10.1109/JSTARS.2015.2446893 that they only can realize ATI baselines shorter than the physical length of the SAR antenna (which is limited by reasons of compactness and weight). Usual ways to create different receive apertures are either to split the SAR antenna electronically into several parts or to cyclically switch the attenuation of subparts along the flight track [4], [5]. For example, the TerraSAR-X satellite utilizes a 4.8-m long antenna. In the so-called dualreceive antenna (DRA) mode, the sensor achieves an effective ATI baseline of only $1.2 \mathrm{~m}$. The consequences are a small time lag and a low sensitivity to ground motions.

The problem can be solved, when the receive apertures in along-track are distributed over spatially separate but cooperating SAR sensors, i.e., when multistatic configurations are applied. With the TanDEM-X (TerraSAR-X add-on for digital elevation measurement) satellite formation, the first such spaceborne multistatic interferometer became available in 2010 [6]. Contrary to the single-satellite case, the mechanical ATI baseline is given here through the spatial separation of the two satellites TSX-1 and TDX-1 of the formation in the flight direction. The sensors are flown on a helix-like orbit that guarantees a safe formation flight and admits wide ranges of adjustment of the satellite's across- and along-track separations as well [7]. Due to the bistatic configuration, there (though possible) is no need to utilize antenna splitting or switching techniques. Compared to single-satellite ATI, this has the advantage that the full SAR antenna can be used for transmitting and receiving and so a higher SNR is achieved. A further advantage of the bistatic data acquisition is that the sensor's full possible Doppler sampling rate $\operatorname{prf}_{\max }$ is available for each receive aperture (for single-satellite ATI, aperture switching leads to an effective $p r f_{\text {eff }}=p r f_{\text {max }} / n_{a p}$, with $n_{a p}$ the number of receive apertures). These properties make TanDEM-X an excellent tool for ocean current imaging. However, there exist also a number of challenges that need to be given consideration, e.g., a hybrid, i.e., combined across-track interferometric (XTI) and ATI baseline, decorrelation over water, phase ambiguities, and absolute phase calibration.

With this paper, we give an overview on TanDEM-X data acquisition and processing for ocean surface observations. Furthermore, we present a variety of results that we have achieved in several studies and where TanDEM-X bistatic ATI has been used to image different sea surface phenomena. This includes tidal currents which could be mapped with unprecedented coverage and resolution at the same time. Furthermore, the high motion sensitivity of the bistatic observations allowed the measurement of slow wind-driven and thermohaline circulations. The interferometric coherence of multistatic spaceborne SAR acquisitions can be an additional 


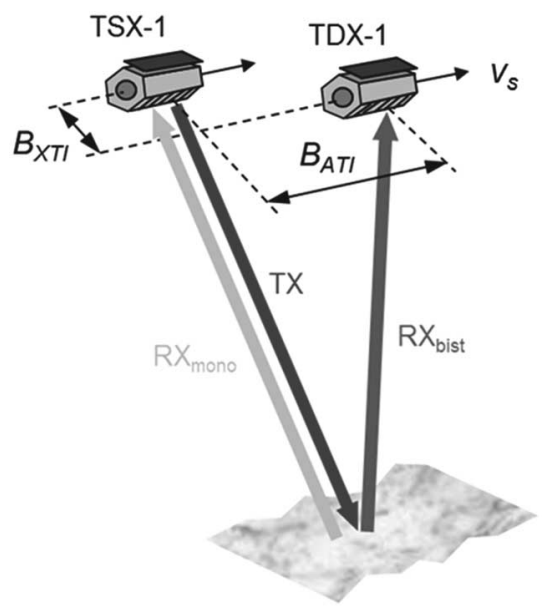

Fig. 1. Principle of bistatic ATI with TanDEM-X.

source of information about the sea surface. Biogenic and anthropogenic changes can alter the appearance of the sea surface in multistatic SAR imagery. We demonstrate on the example of algae blooms how spaceborne SAR systems like TanDEM-X can exploit this to detect and map such phenomena. This paper is structured as follows. We describe TanDEM-X data acquisition and processing aspects relevant for sea surface imaging in Section II. In Section III, we present various results obtained for different application contexts and test sites. Section IV is dedicated to a discussion and in Section V, we draw conclusions and give an outlook on future work.

\section{Methodology}

The two satellites of the formation form a hybrid XT and AT interferometer with the respective mechanical separations or baselines $B_{X T I}$ and $B_{A T I}$ [6]. As TanDEM-X flies on a helical orbit, the relative positions of the two sensors and thus, the baseline components change predictively over time. Practically, this poses the question, for which orbit positions and acquisition times, i.e., for which ground areas surface current mapping is possible under a given configuration or, equivalently, how the formation needs to be adjusted to ensure suitable acquisition geometries [7].

\section{A. Data Acquisition}

In the bistatic mode, one of the sensors is transmitting radar pulses (TX), while both sensors are simultaneously receiving the echoes (RX) from the ground, one via a monostatic $\left(\mathrm{TX}+\mathrm{RX}_{\text {mono }}\right)$, the other one via a bistatic $\left(\mathrm{TX}+\mathrm{RX}_{\mathrm{bist}}\right)$ path of wave field propagation, cf., Fig. 1. The separation of the two sensors along the flight track defines the ATI baseline $B_{A T I}$, the one perpendicular to it is the across-track baseline $B_{X T I}$. The bistatic receive phase center is located at the midpoint of the baseline vector between the two sensors, thus the effective ATI baseline is $B_{A T I, \text { eff }}=B_{A T I} / 2$. The time lag between the mono- and bistatic image is $\Delta t=B_{A T I} /\left(2 \cdot v_{s}\right)$. While the sensors are advancing on a helix-shaped orbit, both baselines components are changing. For instance, $B_{A T I}$ can
TABLE I

TANDEM-X ACQuisition And Processing Parameters for the Data USED IN THIS WORK

\begin{tabular}{llc}
\hline \hline Sensor velocity & $v_{S}$ & $7680 \mathrm{~m} / \mathrm{s}$ \\
Radar wavelength & $\lambda$ & $0.0311 \mathrm{~m}$ \\
Range bandwidth & $B_{r g}$ & $100-150 \mathrm{MHz}$ \\
Azimuth sampling frequency & $p r f$ & $3470-3670 \mathrm{~Hz}$ \\
Processed Doppler bandwidth & $B_{\text {proc }}$ & $2765 \mathrm{~Hz}$ \\
Polarization & & $\mathrm{VV}$ \\
\hline \hline
\end{tabular}

experience a large variation of about $1 \mathrm{~km}$ from one equatorial crossing to the next. The basic TanDEM-X acquisition parameters for the experiments described below are summarized in Table I.

\section{B. Influence of Baseline Components on Sea Surface Observation}

The TanDEM-X baseline vector is generally composed of an ATI and an XTI component. Consequently, the interferometric phase is simultaneously influenced by ground motion and topography. For data acquisitions of the sea surface, the hybrid nature of the interferometer must be taken into account. The motion- and topography-related contributions $\phi_{A T I}$ and $\phi_{X T I}$ add up to the observable interferogram phase

$$
\phi_{i f g r m}=\phi_{A T I}+\phi_{X T I}
$$

with

$$
\phi_{A T I}=\frac{4 \pi}{\lambda} \frac{B_{A T I, \text { eff }}}{v_{s}} \sin \vartheta \cdot v
$$

$$
\phi_{X T I}=\frac{4 \pi}{\lambda} \frac{B_{\perp}}{R_{0} \cdot \sin \vartheta} \cdot \Delta h
$$

Here, $\lambda, R_{0}, B_{\perp}, \vartheta, \Delta h$, and $v$ are the radar wavelength, the slant-range, the perpendicular effective XTI baseline, incidence angle, elevation change, and the observed ground velocity in sensor look direction, respectively. Due to the bistatic data acquisition, $B_{A T I, \text { eff } f}$ and $B_{\perp}$ account for half of the geometric distances. Due to (1), an elevation change must be regarded as an error, when the velocity is estimated from $\phi_{\text {ifgrm }}$. Although the topographic interferometric phase of the ocean surface can be regarded as constant after compensation of the flat earth's contribution, height variations might locally occur, essentially through waves. Setting (2) equal to (3), rearranging after $v$ and setting $\Delta h=1 \mathrm{~m}$ yields the error of the estimated sea surface velocity equivalent to 1 -m elevation

$\Delta v_{1 m \text { - equivalent }}=\frac{B_{\perp} \cdot v_{s}}{B_{A T I, \text { eff }} \cdot R_{0} \cdot \sin ^{2} \vartheta} \Delta h$ for $\Delta h=1 \mathrm{~m}$.

Assuming typical baseline components of $B_{\perp}=40 \mathrm{~m}$ and $B_{A T I, \text { eff }}=25 \mathrm{~m}$, Table II shows the 1 -m-elevation equivalent velocity error for different incidence angles.

The influence of the wave heights on the interferometric phase increases toward smaller incidence angles. Practically, the choice of $\vartheta$ depends on different factors. While smaller incidence angles are favorable in terms of higher backscatter and 
TABLE II

One-Meter-Elevation Equivalent Velocity ERror For Bistatic BASELINES OF $B_{\perp}=40 \mathrm{~m}$ AND $B_{A T I, \text { ef } f}=25 \mathrm{~m}$

\begin{tabular}{ccc}
\hline \hline$\vartheta\left({ }^{\circ}\right)$ & $R_{0}(\mathrm{~m})$ & $\Delta v_{\text {lm-equivalent }}(\mathrm{m} / \mathrm{s})$ \\
\hline 25 & 564114 & 0.121 \\
35 & 614517 & 0.060 \\
40 & 660394 & 0.045 \\
\hline \hline
\end{tabular}

better SNR, larger angles allow for higher sensitivity to ground motions. Medium incidence angles of e.g., $35^{\circ}$ provide a good compromise. The remaining error due to wave height variations on the sea surface can be reduced further when the interferometric phase or, equivalently, the velocity estimated from it, is spatially averaged over several resolution cells.

Another important aspect to consider is the choice of the ATI baseline. Larger values of $B_{A T I}$ are desirable in order to maximize the sensitivity to ground motion. However, for the multistatic radar, i.e., for its two successive observations, the radar signal backscattered from the sea surface will decorrelate after the coherence time $t_{c}$ (which depends essentially on the surface wind speed and on the effective imaging resolution) [8], [9]. Therefore, the time lag $\Delta t$ must not exceed $t_{c}$ and the upper limit for the ATI baseline is given according to $B_{A T I, \text { eff }}<$ $v_{s} \cdot t_{c}$. Assuming a value of $t_{c}=5 \mathrm{~ms}$ in X-band and for $10 \mathrm{~m} / \mathrm{s}$ wind speed, $B_{A T I, \text { eff }}$ should be less than $38.4 \mathrm{~m}$. It is worth noting here that multistatic SAR configurations offer an elegant technical means to estimate the autocorrelation function of the backscattered radar signal from sea surface and $t_{c}$. This can be done by analyzing scene coherence values for a larger amount of data acquisitions and different ATI baselines. First results with TanDEM-X have been presented in [10] and [11].

Since $\phi$ is bound to the interval $[-\pi, \pi]$, a too large $B_{A T I}$ can also result in phase wrapping and in ambiguous estimates of the ground velocity. The unambiguously measurable ground velocity range accounts to $-V O A / 2 \leq v<V O A / 2$ with $V O A$ being the velocity-of-ambiguity

$$
V O A=\frac{\lambda \cdot v_{s}}{2 \cdot B_{A T I, \text { eff }} \cdot \sin \vartheta} .
$$

\section{Data Processing}

To retrieve information on sea surface motions from the bistatic interferometric data, we use a two-step processing chain, which consists of the operational integrated TanDEM-X processor (ITP) and a dedicated postprocessing system. The ITP was developed for the primary TanDEM-X mission goal of producing a highly precise global DEM [12]. It creates several intermediate data products during processing. The so-called CoSSC data product consists of the two coregistered, single-look complex images that correspond to the monoand bistatic TanDEM-X data acquisition channel. This data set is fed in our postprocessing chain for surface current extraction, which is shown in Fig. 2. After interferogram formation, multilooking and removal of the phase caused by the flat earth, phase unwrapping using a minimum cost flow (MCF) algorithm is performed to remove possible phase ambiguities [13]. Considering the high sensitivity of the

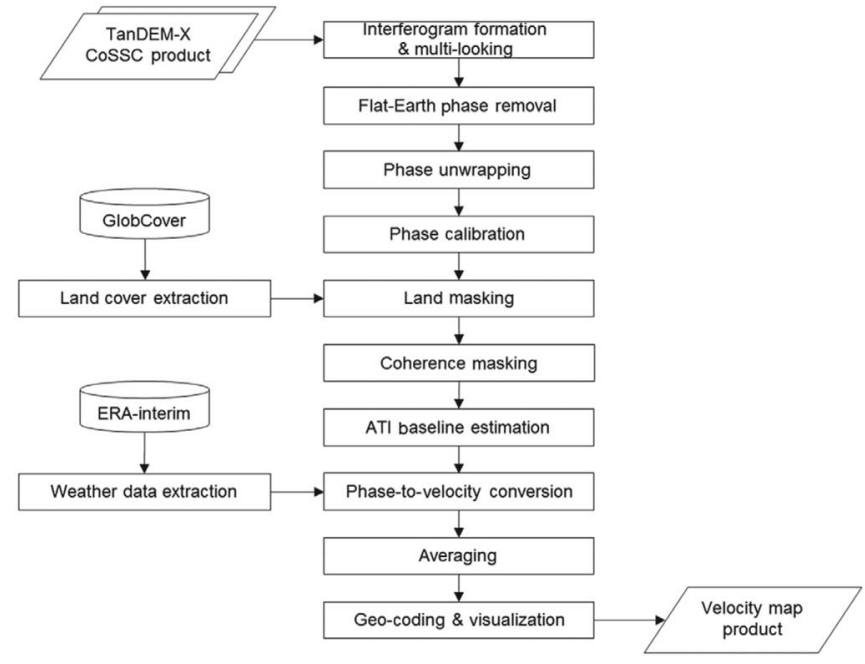

Fig. 2. Flowchart of the surface current extraction processor for TanDEM-X CoSSC data.

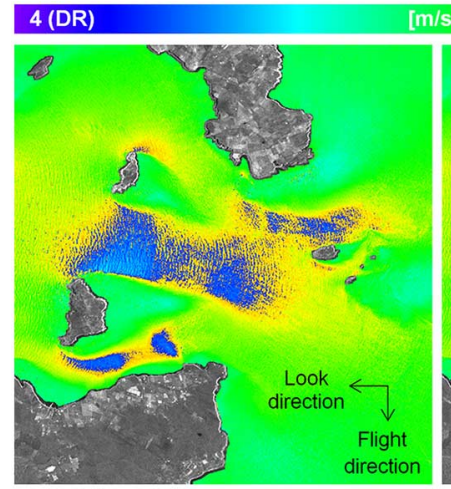

(a)

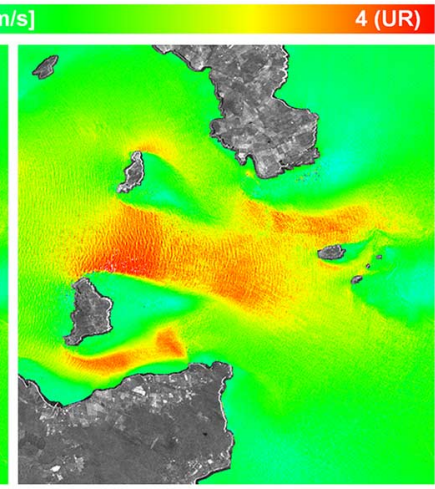

(b)
Fig. 3. Velocity maps derived from (a) an uncorrected and (b) a corrected, i.e., unwrapped interferogram phase of a TanDEM-X data take of a tidal current.

TanDEM-X AT interferometer, even slow ground motions can cause the interferometric phase to be wrapped. For example, a configuration with $B_{A T I, \text { eff }}=25 \mathrm{~m}$ and $\vartheta=40^{\circ}$ resulted in $\mathrm{VOA} / 2=3.6 \mathrm{~m} / \mathrm{s}$. A ground velocity of $4 \mathrm{~m} / \mathrm{s}$, which can occur for tidal currents, caused a measured phase that is ambiguous by one cycle, thereby pretending a ground velocity of $-3.2 \mathrm{~m} / \mathrm{s}$. Thus, it is necessary to unwrap the interferogram phase prior to converting it into velocities. Fig. 3(a) and (b) shows examples of velocity maps (in slant-range projection) derived from an uncorrected and a corrected, i.e., unwrapped TanDEM-X interferogram of the Pentland Firth, Orkney Islands, U.K. Land areas are replaced by the SAR amplitude. There is a surface current toward the sensor, i.e., in up-range (UR) direction, recognizable by the yellow color. In the uncorrected case (a), implausible jumps of the flow to the opposite direction (blue) are visible, which correspond to untreated phase ambiguities. In case (b), the error has been corrected through the phase unwrapping step applied to the measured wrapped phase. As a result, a consistent flow field is obtained (yellowish and reddish colors represent the same direction).

Phase calibration is performed interactively by estimating the mean phase in coastal areas that can be assumed to be on sea 
level and that are not severely influenced by wave and currents (bays, ports etc.). These areas should have a phase bias, which is caused by the elevation of the sea surface and by processing offsets, but not by surface motion. In the following, we assume that a correction for the interferometric phase caused by the flat earth (modeled through a WGS84 ellipsoid) has been applied. Due to the topographic influence on the interferometric phase, the local difference of the sea surface to the ellipsoid can cause a phase offset as can the phase unwrapping step. For a pure XTI system, the bias could be determined by using a set of ground control points (GCP) with known elevation (or phase, equivalently). However, this is not applicable over the open sea for an ATI/XTI system as the phase induced by surface currents would be misinterpreted as an error and be compensated. Calibration has therefore to be carried out over land areas using a DEM or in coastal areas with the constraint that the interferogram phase and surface current velocity associated with it must be zero. Using a DEM or GCP over land has the advantage that it can be carried out automatically. However, this is difficult as elevation data of very precise absolute height are required after (4). Calibration by estimating the phase offset in coastal areas does not require such additional data, but has to be done semi-automatically in return.

Land areas are masked out in the next step by applying a land mask, which is extracted from the GlobCover data base [14] and converted into slant-range projection. As incoherent water areas correspond to high phase noise and will lead to significant errors in the velocity maps generated later-on, we apply a further masking based on comparing the magnitude of the interferometric coherence against an empirical threshold. Before the calibrated phase is converted into a velocity map by applying (2) rearranged after $v$, the effective ATI baseline is estimated. We take into account the temporal changing of $B_{A T I}$ in the phase-to-velocity conversion. Using a fixed (average) value would lead to significant velocity errors, especially for longer data takes.

At this point, also unwanted wind-induced contributions to the measured velocity can be compensated. An automatic weather data extraction has been integrated in the processor, in order to retrieve 10-m-height wind vectors from a local ERA-Interim data base [15]. As the spatial variation of the wind influence can be severe for the large areas imaged with the TanDEM-X data takes, a locally adaptive approach is required for compensation. Currently, the wind information is not yet used automatically by the processing system, but we have planned the implementation of a geophysical inversion model like the one proposed by [9]. However, the automatically extracted wind vectors already aid in interpreting the obtained velocity maps.

The velocity is averaged to improve the SNR (of course at the cost of losing some spatial resolution). Finally, the velocity map as well as the interferometric phase and coherence are geo-coded to create a visualization product, which includes e.g., KML files for GoogleEarth.

\section{RESUltS}

Within the scope of different studies, we have processed TanDEM-X data of a larger amount of ocean test sites, either using acquisitions made during dedicated ocean application campaigns or acquisitions of opportunity. The results obtained demonstrate the enormous potential that space based bistatic ATI has for ocean applications.

\section{A. Tidal Current Mapping}

Tidal currents are water flows, which are caused by the gravitational forces of moon and sun. Beside their relevance for navigation, tidal currents have gained huge interest in the past years in the context of renewable energy i.e., for the installation of power plants that convert tidal into electrical energy [16]. To find suitable sites and to evaluate their energetic potential are key questions in this context and rely on large-area flow measurements. Since 2012, we have been using TanDEM-X ATI to image tidal currents in the Pentland Firth Sound, North of the Scottish mainland, U.K. This region is an excellent test site as it features large-area tidal flows that reach velocities of up to $7 \mathrm{~m} / \mathrm{s}$. This was shown in previous works using SRTM, TerraSAR-X and TanDEM-X [17], [18]. Although SRTM had a 7-m along-track baseline, which allowed for a moderate sensitivity to ground motion, the 11-day mission was not designed for frequent oceanographic observations. With TerraSAR-X, the first long-term spaceborne SAR sensor with ATI became available. However, its short ATI baseline makes it only suboptimal for surface current mapping. In contrast to that TanDEM-X admits both frequent and highly sensitive measurements of ocean surface motion phenomena at the same time. Fig. 4 shows results from three different TanDEM-X data takes over the Pentland Firth on March 14, 2012 (a), August 22, 2014 (b), and September 01, 2014 (c). All data takes were acquired from descending orbit passes with the sensor look direction to the right. The images are georeferenced and represent the wrapped, uncorrected phase, the interferometric coherence, and the ground velocity in the sensor look direction (from left to right) obtained after applying the described processing. Land has been replaced in the phase and in the velocity images by the SAR amplitude of the monostatic channel to ease visual interpretation. Furthermore, note that areas of low SNR have been masked out in the velocity images for coherences $\gamma<0.4$. The color coding of the velocity images is such that bluish colors indicate a motion in down-range (DR) direction, i.e., away from the sensor, reddish colors a motion in UR direction, i.e., toward the sensor. For the March 14, 2012 case, the TanDEM-X velocity map shows a strong tidal flow from SE to NW. Notice how the map reflects that the flow is circumventing the two small Islands of Stroma and Swona within the Sound with several branches that join into one main flow behind them. In the areas between the flow branches, countercurrents or eddies are generated, which are recognizable by the yellowish blobs. The time of acquisition was almost coinciding with the occurring of the maximum flow speed between two subsequent vertices of the tidal cycle. On August 22, 2014, a tidal flow in the opposite direction was imaged by TanDEM-X. In the third case (September 01, 2014), a moderate flow to the NW was imaged, visible by the bluish colors. Note how "flow shadows" develop on the respective far sides of the two small Islands within the sound. Table III lists specific acquisition parameters together with wind data. The large incidence angles 
(a)

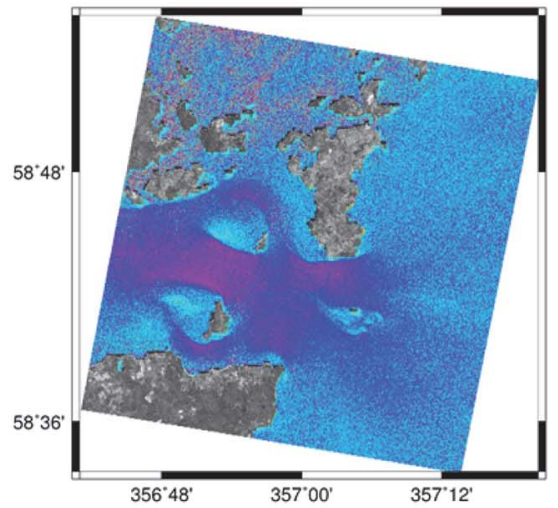

(b)

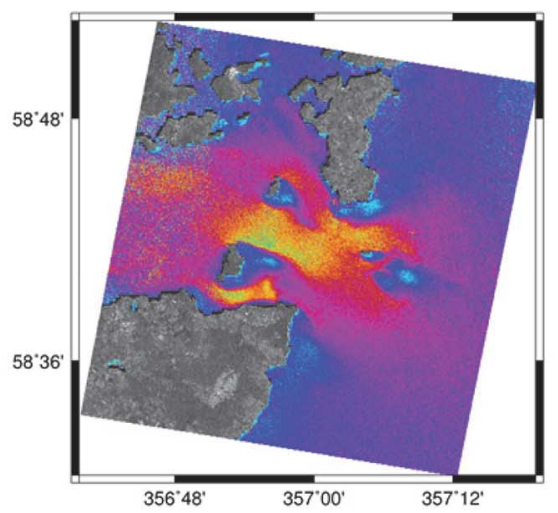

(c)

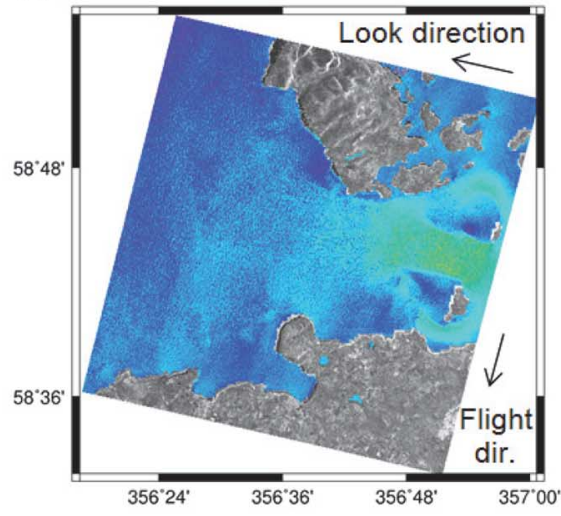

$-\pi$

Wrapped phase
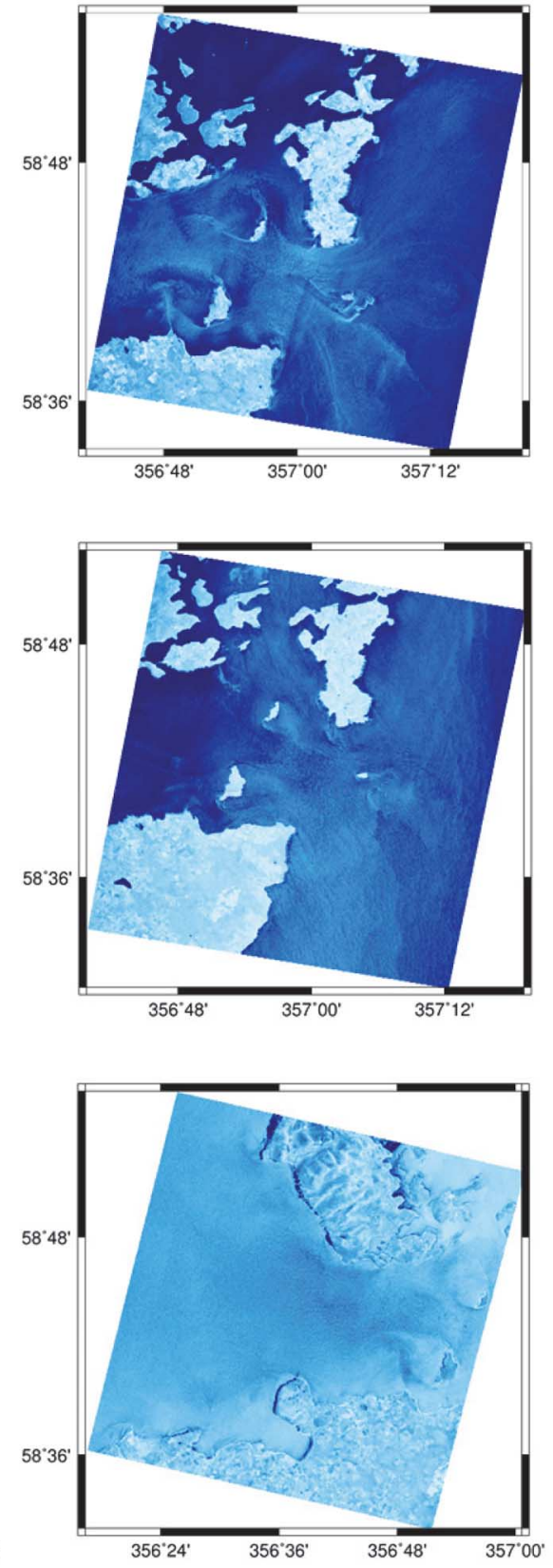

0.0
Ground-range velocity
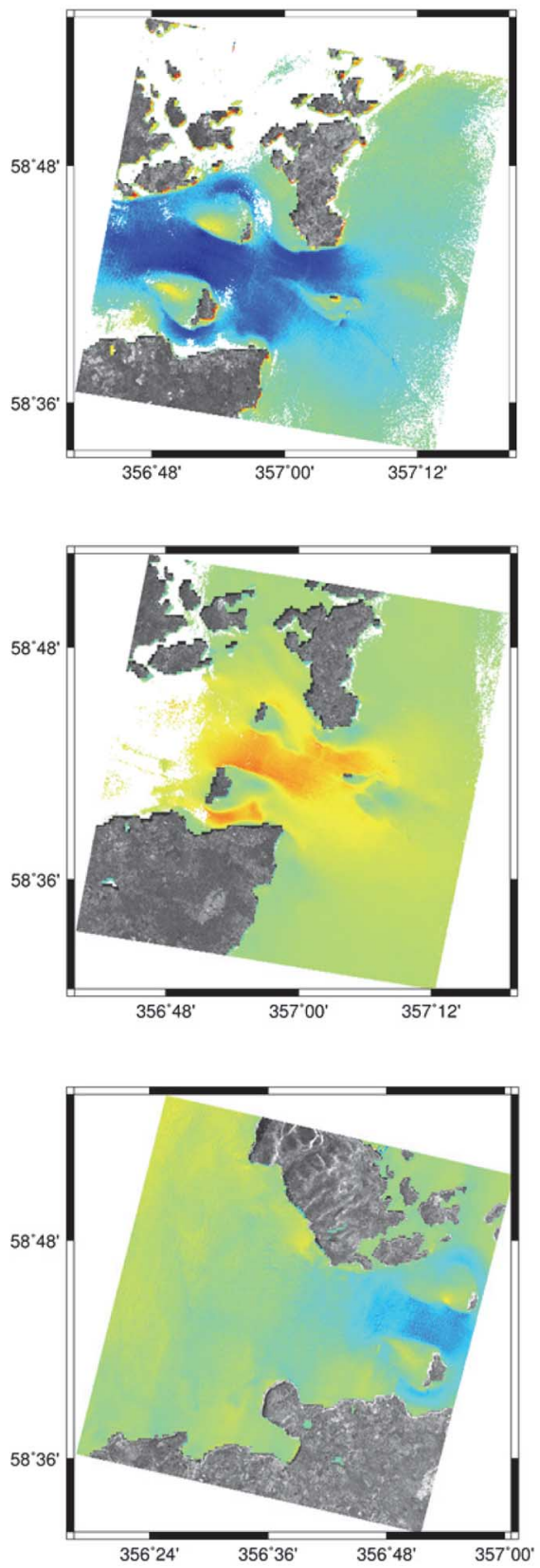

7 (DR) $\quad(\mathrm{m} / \mathrm{s}) \quad 7$ (UR)

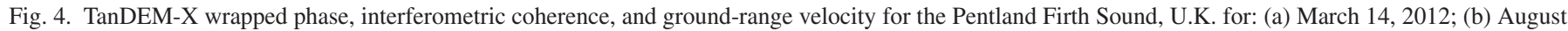

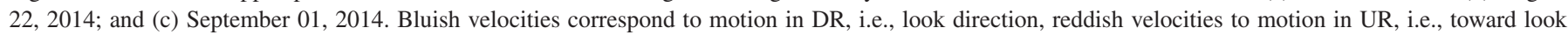
direction.

in the first two cases cause some decrease in the backscatter and lead to image regions of low coherence. Due to that, some parts in the Northwest of these scenes were considered unreliable by the processor and masked out. However, the main regions with the tidal flow were captured. In the third case, the small incidence angle leads to increased backscatter on the sea surface and the coherence is very high throughout the complete scene.

Among the several sites, where we have tested our methodology of tidal current mapping is also the Bay of Fundy, Canada.
This region features strong tidal flows and has recently been considered for installation of a tidal energy power plant [19]. Being part of a special TanDEM-X campaign with short ATI baselines for ocean imaging in the northern hemisphere in summer of 2014, we could acquire bistatic ATI data over this site on August 20. The data take was made from a descending orbit, right-looking at an incidence angle of $\vartheta=48.1^{\circ}$ and with an effective ATI baseline of $B_{A T I, \text { eff }}=18.3 \mathrm{~m}$. The respective wrapped phase (a), interferometric coherence (b), and velocity 
TABLE III

TANDEM-X ACQUisition PARAMETER AND Weather For PENTLAND FIRTH TEST Sites

\begin{tabular}{lcccc}
\hline \hline UTC acquisition time & $\begin{array}{c}\text { Effective } \\
\text { ATI } \\
\text { baseline }(\mathrm{m})\end{array}$ & $\begin{array}{c}\text { Incidence } \\
\text { angle }\left({ }^{\circ}\right)\end{array}$ & $\begin{array}{c}\text { Wind speed } \\
(h=10 \mathrm{~m}) \\
(\mathrm{m} / \mathrm{s})\end{array}$ & $\begin{array}{c}\text { Wind } \\
\text { direction } \\
(h=10 \mathrm{~m})\left({ }^{\circ}\right)\end{array}$ \\
\hline 2012-03-14,06:32:46 & 6.8 & 41.4 & 5.7 & 204 \\
2014-08-22,06:33:04 & 30.1 & 41.4 & 8.9 & 335 \\
2014-09-01,06:50:12 & 26.5 & 21.6 & 6.8 & 252 \\
\hline \hline
\end{tabular}

image (c) are shown in Fig. 5. Although some ocean areas in the Western part and at the coasts of the scene were blanked out by the algorithm due to a low SNR $(\gamma<0.4)$, a large part of the tidal flow at the acquisition time was captured and is recognizable by the reddish colors. They indicate a flow that is directed to the East, i.e., into to Bay. This corresponds to what is expected, as the Bay was imaged by TanDEM-X at 10:21 UTC, i.e., during the transition from low tide (06:23 UTC) to high tide (12:28 UTC), measured nearby at the "Burntcoat Head" station [20].

\section{B. Ocean Circulations}

Ocean circulations are driven by wind and thermohaline mechanisms. The Gulf Stream is one of the most prominent examples. Since these phenomena imply transports of water, heat, and salt, they are tightly associated with climate variability, thus, their monitoring is of great importance [21]. Although circulations also manifest as surface currents and are observable by remote sensing techniques, their velocity is relatively slow (almost one order of magnitude less than tidal currents). Here, spaceborne multistatic SAR ATI with its high motion sensitivity can significantly contribute to retrieve information on this type of processes.

A first case, where we could image an ocean circulation with TanDEM-X data and extract the associated surface velocity, is shown in Fig. 6. On February 26, 2012, TanDEM-X imaged the Le Maire Strait between the Argentinean mainland and the Isla de los Estados at an incidence angle of $26.5^{\circ}$ and with an effective ATI baseline of $26.5 \mathrm{~m}$. From South to North, the data take spans a distance of about $110 \mathrm{~km}$. The interferometric phase (a) exhibits some interesting variations over water, which result in the respective ground velocity image (b). This shows a large area, where velocities are directed in DR direction of the sensor, i.e., to the East. These surface velocities, shown in yellowish to reddish colors, are most likely associated with a branch of the circumpolar current [22].

In 2012, we started a project were surface currents in the Baltic Sea are imaged with TanDEM-X. It is part of a comprehensive study called "Remote Sensing and Earth System Dynamics," which aims at deriving climatological relevant information from spaceborne SAR within the geosphere, biosphere, cryosphere and hydrosphere [23]. In this part of the hydrosphere section remote sensing data, models and in situ measurements shall be combined. As an example, Fig. 7 shows a surface current imaging result from the Baltic Sea, South of Trelleborg, Sweden. Data were acquired at an incidence angle of $\vartheta=29.9^{\circ}$ and with an effective ATI baseline of

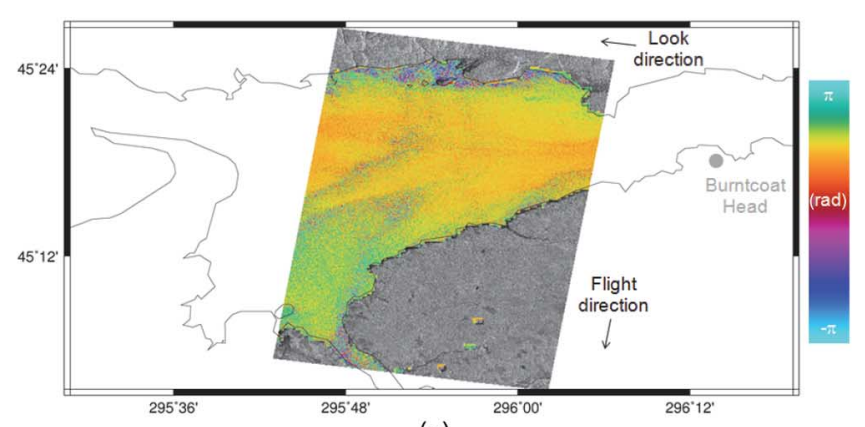

(a)

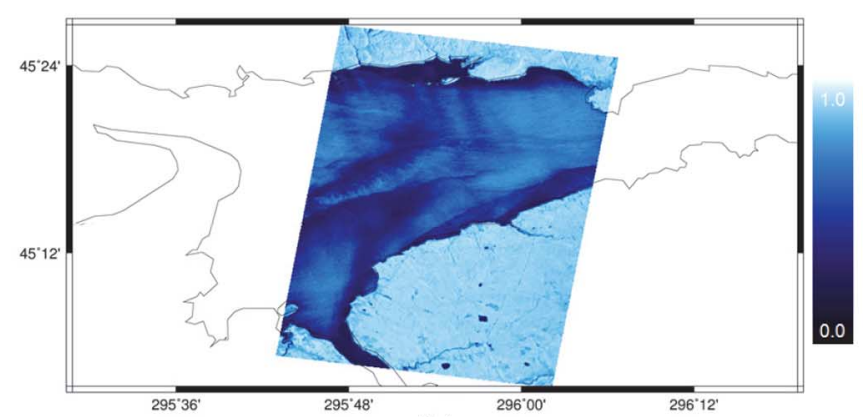

(b)

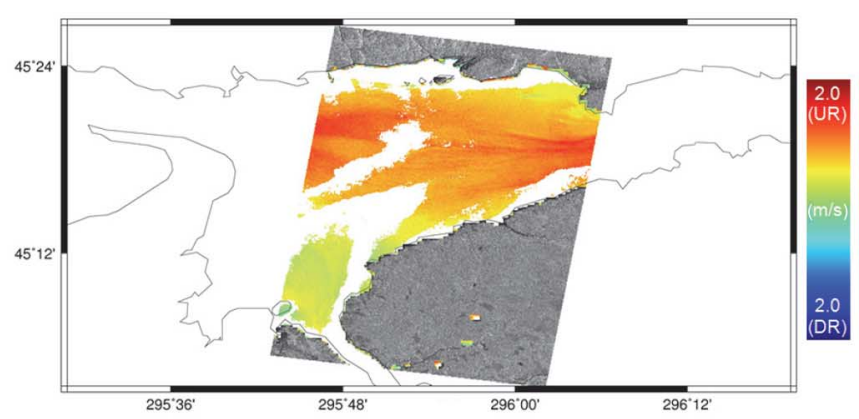

(c)

Fig. 5. (a) TanDEM-X wrapped phase, (b) interferometric coherence, and (c) extracted ground velocity of August 20, 2014, 10:21 UTC for a part of the Bay of Fundy, Canada.

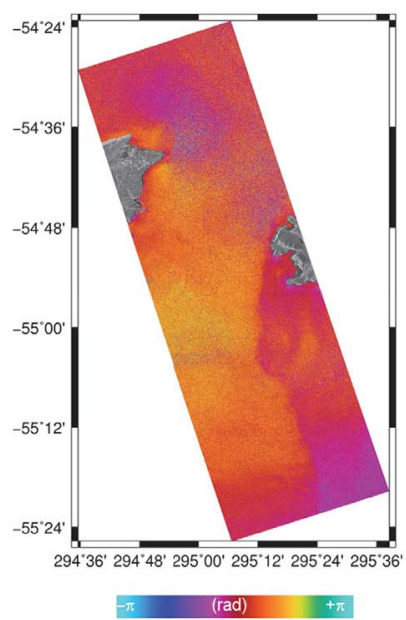

(a)

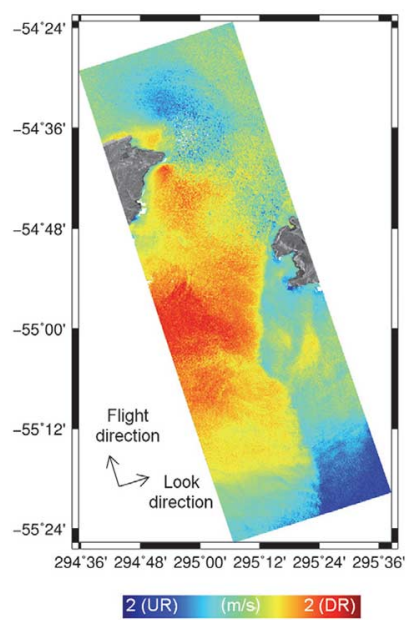

(b)
Fig. 6. (a) TanDEM-X bistatic interferometric phase and (b) extracted ground range velocity for the Le Maire Strait, Argentina on February 26, 2012. 


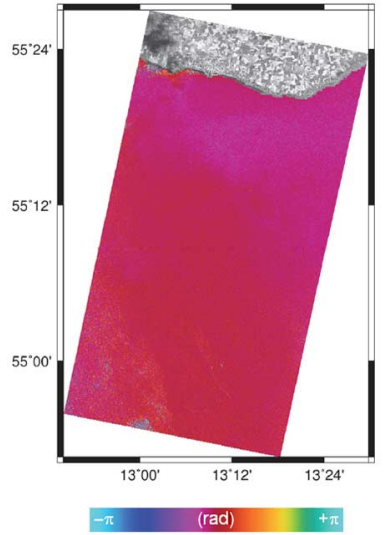

(a)

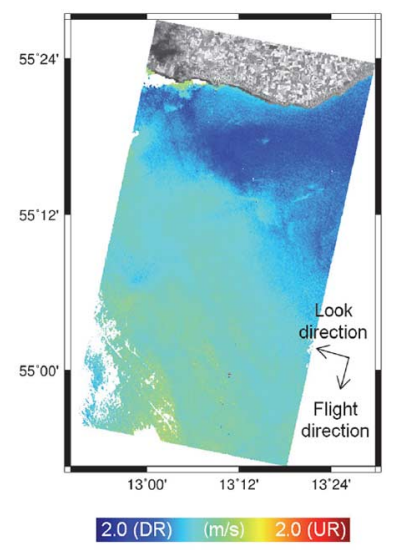

(b)
Fig. 7. (a) TanDEM-X bistatic interferometric phase and (b) extracted ground range velocity for the area South of Trelleborg, Sweden, August 31, 2014.

$B_{A T I, \text { eff }}=12.9 \mathrm{~m}$. In the velocity image (b), a wind-driven surface current toward the West is recognizable (bluish colors). This is in correspondence with the $4.4 \mathrm{~m} / \mathrm{s}$ southeastern wind $\left(121^{\circ}\right)$ that we extracted from ERA Interim for the acquisition time.

\section{Detection and Mapping of Surface Films}

Another interesting oceanographic application of multichannel spaceborne SAR systems lies in the detection and mapping of biogenic and anthropogenic surface films, e.g., alga blooms or waste deposits. Several works showed that the drift of sea surface films can be measured from space using repeated and/or intersatellite image acquisitions with time lags of several minutes to hours and correlation techniques that enable to track patterns on the sea surface [24], [25]. Spaceborne bistatic SARATI offers a new and effective way to image and map these phenomena with a single data acquisition. It is known that surface films or slicks change the radar backscatter of the sea surface. This leads to the question, how the ATI phase and the interferometric coherence of a bistatic SAR are influenced and if they can be used for detecting areas with occurrence of such films. We looked at TanDEM-X acquisitions of oceanic regions which are known to have frequent occurrences of alga blooms. The Baltic Sea is one such area, where the phenomenon can be observed during summer. On June 28, 2011, a TanDEM-X acquisition North of the Island of Gotland, Sweden was made at an incidence angle of $\vartheta=26.5^{\circ}$ and an effective ATI baseline of $B_{A T I, \text { eff }}=100 \mathrm{~m}$. It revealed interesting features after data processing, cf., Fig. 8 . The amplitude image (a) exhibits typical patterns of algae on the water surface. The substances released by the algae cause a reduced radar backscatter compared to the uncovered, open water areas. Weather data extracted for the time of the TanDEM-X acquisition show a slow wind speed of $u_{10}=1.3 \mathrm{~m} / \mathrm{s}$. This leads to relatively low roughness and backscatter of the water surface, but the effect of local backscatter reduction through the algae is still recognizable. The coherence image is shown in Fig. 8(b). Although the time lag between the mono- and the bistatic image acquisition was relatively long $(13 \mathrm{~ms})$, the coherence for the open water areas is high. We explain this by the low wind speed, which, for the given radar frequency and image resolution and under the assumption of a Pierson-Moskowitz-type wave spectrum, yields a predicted coherence time in the order of $70 \mathrm{~ms}$ [26]. Both the coherence and the amplitude assume a bimodal distribution in case surface films are present. Note that the contrast between the areas with and without the alga surface film is more pronounced in the bistatic interferometric coherence than in the amplitude image. Thus, the interferometric coherence obtained with multistatic SAR ATI sensors can provide a means for discrimination between areas covered and those not covered by certain kinds of surface films. We have tested this methodology by integrating a segmentation algorithm, which utilizes a simple binary classification, into the processing chain. Based on variance criteria, it automatically searches for a threshold, which divides image pixel values into two optimally separated classes. Details of the algorithm can be found in [27]. We applied this classification on the coherence and on the amplitude image of the Baltic Sea data set of Fig. 8. The separability criterion $\eta\left(T_{o p t}\right)=\sigma_{b}^{2}\left(T_{\text {opt }}\right) / \sigma_{a}^{2}$ as defined in [27] with $0 \leq \eta \leq 1$ and $T_{\text {opt }}, \sigma_{b}^{2}\left(T_{\text {opt }}\right)$ and $\sigma_{a}^{2}$ being the found optimal threshold, the between-class variance and the all-pixel variance, was used to compare the quality of the classifications. These resulted in separability measures of $\eta_{A}=0.617$ and $\eta_{\gamma}=0.779$ for the amplitude $A$ and the coherence $\gamma$, respectively. This means that the coherence image permits better discrimination between the two classes than the amplitude image.

The classification result based on the coherence image is shown in Fig. 8(d). By applying an automatically determined threshold $T_{o p t, \gamma}=0.42$ to the coherence and by improving the consistency of the image regions obtained this way through morphological erosion and dilation operators, a segmentation mask was derived. The mask indicates areas with and without algae blooms. Since both surface types are spatially interwoven and since open water shows high coherence, it becomes even possible to estimate the drift velocity of the whole area including the algae patches from the interferometric phase image of Fig. 8(c). A map of the open water areas (which enclose the algae patches), color-coded after their velocity, is shown in Fig. 9. The bluish colors indicate a slight drift component in the UR direction of the sensor, i.e., to the West. This is consistent with the wind direction extracted from the ERA Interim data, which was $150^{\circ}$, i.e., from SE.

\section{Discussion}

Our experiments show that different phenomena of the sea surface can be detected and quantified by using space-based dual-channel SAR ATI. The time lag between the receive apertures of the sensor is one of the most critical parameters and determined by the ATI baseline. For the measurement of motions, it has to be shorter than the decorrelation time of the radar signal backscattered from the water surface. It has been shown for TanDEM-X that desired ATI baselines could be adjusted for certain latitudes on a daily basis [7]. However, this approach can only be followed in short campaigns, because this adjustment alters the XTI baseline too, which might compromise other TanDEM-X missions objectives. 


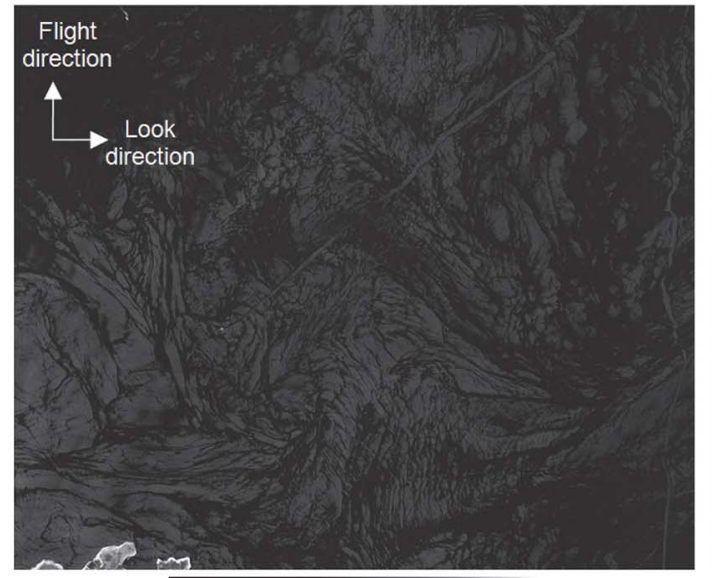

(a)

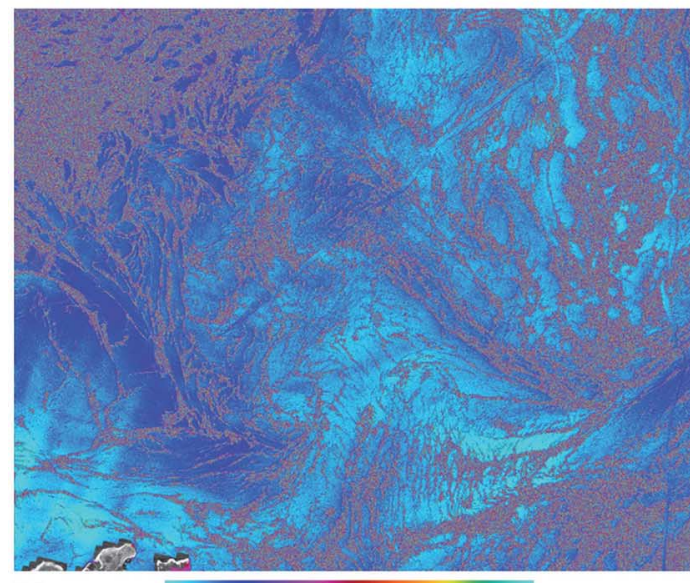

(c)
0

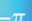

(rad)

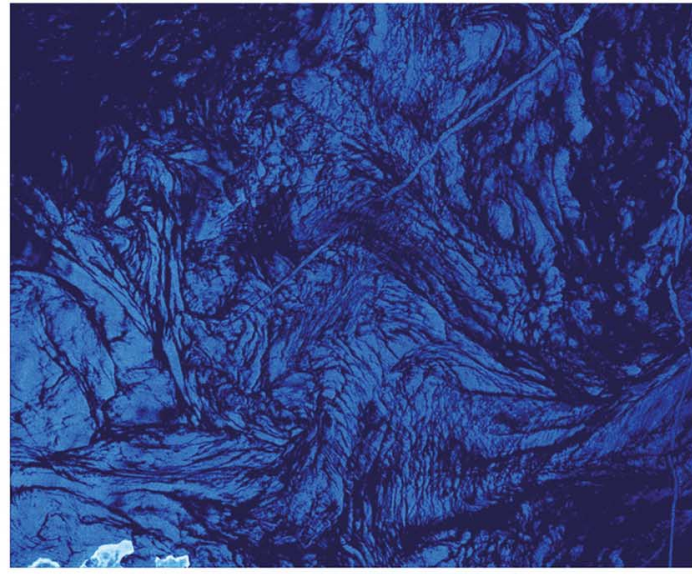

(b)

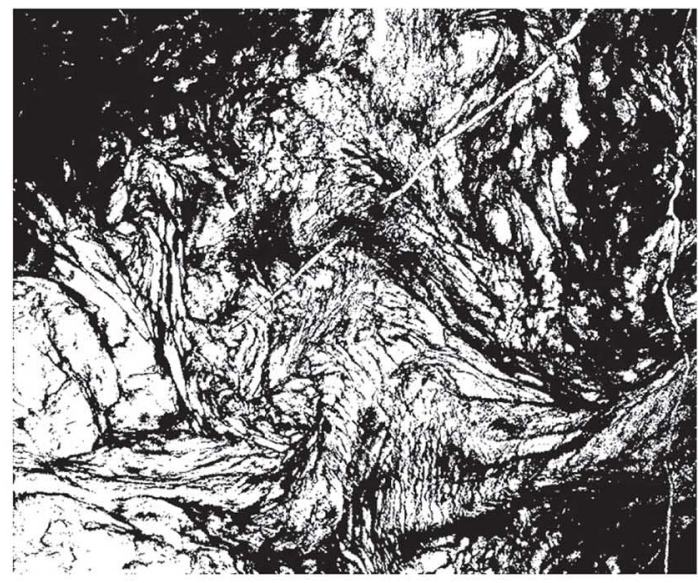

(d)

Fig. 8. Part of TanDEM-X bistatic data of an area with alga blooms in the Baltic Sea near Gotland, Sweden, June 28, 2011. (a) SAR amplitude, (b) interferometric coherence, (c) phase, and (d) the classification mask derived from coherence in slant-range projection.

With the described method, the LOS component of the surface currents is measured. For many cases, this will be sufficient as the two-dimensional (2-D) flow direction is known from models or in case of wind-driven currents can be estimated from supplementary (weather) data or from the wave structure and backscatter in the SAR images itself.

For the case of detecting areas that enclose certain sea surface films like alga blooms the interferometric coherence can provide a suitable means. Water areas that are not covered by those films show normal coherence, thereby giving a high contrast to areas covered by the film, which appear decorrelated in the interferogram. Of course, there exists an upper limit for the useful ATI time lag also in this application. It has to be shorter than the mentioned decorrelation time in order to obtain high coherence for the open-water areas, i.e., good contrast to the decorrelated areas covered by the surface films. Furthermore, moving scatterer must not leave resolution cells in between the image acquisitions of the two channels. Finally, if drift velocities are estimated from the ATI phase in the coherent areas, a too high sensitivity might cause multiply wrapped phase values, which can be difficult to reconstruct. The last two points also apply to all applications shown here. The described method works excellent for surface films like alga blooms, which show a large spatial extent. For other kinds of films like oil spills, the methodology could be suited as well but has probably to be adapted.

Although being beyond the scope of this work, we would like to state that for geophysical interpretation, the extracted velocity fields need to be calibrated for wind and wave effects, depending on the type of application. Wind-driven surface motion should e.g., be compensated in the case of tidal current measurements. Such an effect is visible in the velocity image of Fig. 4(c). It indicates the presence of two super-imposing effects-a strong tidal flow from East to West (bluish colors on the right side of the image) and a slight wind-driven surface motion directed in the opposite direction (light yellowish colors visible on the left side), an observation, which is supported by the extracted wind data $\left(6.8 \mathrm{~m} / \mathrm{s}\right.$ from $252^{\circ}$, i.e., from the West). Implementing a local adaptive calibration scheme will be one of the next steps in the further development of the surface current data processor.

\section{CONCLUSION AND OUTLOOK}

The presented TanDEM-X results demonstrate the great potential that multistatic spaceborne SAR has for 


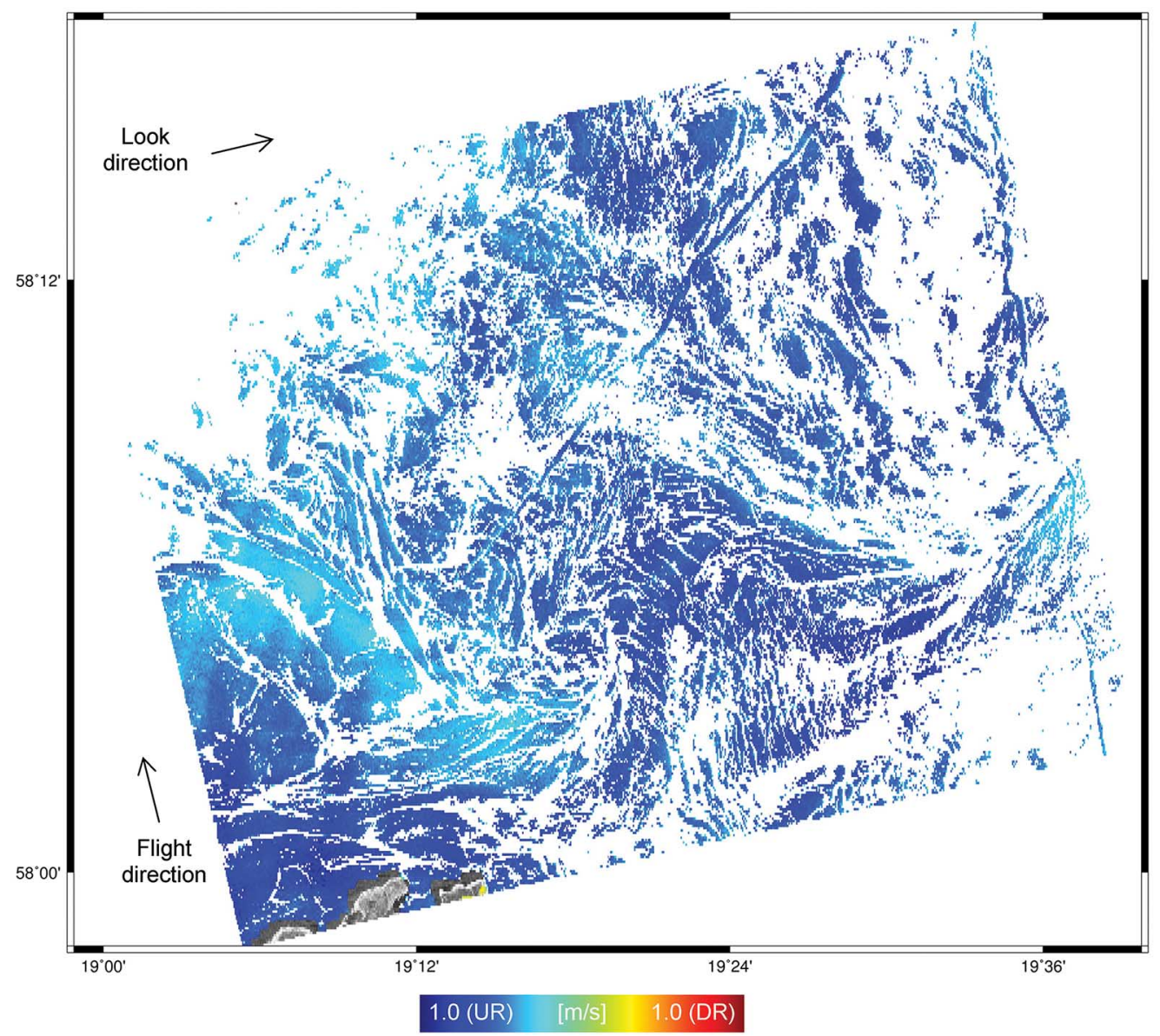

Fig. 9. Surface drift velocities of an area with algae bloom patterns automatically detected and estimated in TanDEM-X bistatic data acquired on June 28,2011 , North of Gotland, Sweden.

oceanography. Tidal currents and ocean circulations could be measured with very high sensitivity. Because of its ecological value, the measurement of tidal currents is an ideal application for multichannel spaceborne SAR. Furthermore, tidal currents usually appear close to coastal regions. This helps in absolute calibration of the obtained velocity maps. Although thermohaline circulations are widely covered by altimeter satellites, these provide less spatial resolution and have difficulties in coastal regions. Here, multichannel SAR formations like TanDEM-X can be of great advantage. We also showed that areas that contain alga blooms can be detected and that their drift velocity can be estimated.

For this work, we made use of TanDEM-X dual-channel SAR ATI which captures the LOS component of ground motions with respect to the sensor. However, the TanDEM-X formation offers also different possibilities for direct 2-D observations of the sea surface. The first one consists in operating both TanDEM-X satellites in the mentioned DRA mode, where either sensor transmits with the full SAR antenna and receives with two electronically separated halves of it. In addition to that, one or both of the satellites are significantly squinted. This yielded different LOS vectors of the two separately operating ATI systems. Although this four-channel configuration would be less sensitive to ground motion than the bistatic one, it allowed to retrieve different directional components of a surface current, which can be combined to a 2-D measurement [28]. Up to now, no data are available from this mode. The second possibility has recently be described and demonstrated in [29]. It exploits the so-called bidirectional SAR mode, where the SAR antenna creates a pair of symmetrically squinted beams. Applying this on the TerraSAR-X and the TanDEM-X sensor (both still operating in bistatic mode), one slightly forward- and one slightly aft-looking AT interferometer are obtained, which also allow to recover 2-D velocity vectors. Especially the measurement of sea surface film drifts can benefit from 2-D motion measurements. Additionally, also the application of wide-swath imaging modes will be of interest in the future since they allow access to large ocean areas.

\section{ACKNOWLEDGMENT}

The authors would like to thank the reviewers for their valuable comments.

\section{REFERENCES}

[1] R. M. Goldstein and H. A. Zebker, "Interferometric radar measurement of ocean surface current," Nature, vol. 328, no. 6132, pp. 707-709, 1987.

[2] R. Romeiser et al., "Current measurements in rivers by spaceborne along-track InSAR," IEEE Trans. Geosci. Remote Sens., vol. 45, no. 12, pp. 4019-4031, Dec. 2007. 
[3] R. Romeiser, S. Suchandt, H. Runge, U. Steinbrecher, and S. Grünler, "First analysis of TerraSAR-X along-track InSAR-derived currents fields," IEEE Trans. Geosci. Remote Sens., vol. 48, no. 2, pp. 820-829, Feb. 2010.

[4] H. Runge, C. Laux, R. Metzig, and U. Steinbrecher, "Performance analysis of virtual multi-channel TS-X SAR-modes," in Proc. Eur. Conf. Synth. Aperture Radar (EUSAR'06), Dresden, Germany, May 16-18, 2006, 4 pp.

[5] I. Sikaneta, "Demonstrations of HRWS and GMTI with RADARSAT-2," in Proc. 9th Eur. Conf. Synth. Aperture Radar (EUSAR'12), Nürnberg, Germany, Apr. 23-26, 2012, pp. 263-266.

[6] G. Krieger et al., "TanDEM-X: A satellite formation for high-resolution SAR interferometry," IEEE Trans. Geosci. Remote Sens., vol. 45, no. 11, pp. 3317-3341, Nov. 2007.

[7] R. Kahle, H. Runge, J.-S. Ardaens, S. Suchandt, and R. Romeiser, "Formation flying for along-track interferometric oceanography-first inflight demonstration with TanDEM-X," Acta Astronaut., vol. 99, pp. 130142, 2014.

[8] W. J. Plant, E. A. Terray, R. A. Pettit, Jr., and W. C. Keller, "The dependence of microwave backscatter from the sea on illuminated area: Correlation times and lengths," J. Geophys. Res., vol. 99, no. C5, pp. 9705-9723, 1994.

[9] R. Romeiser and D. R. Thompson, "Numerical study on the along-track interferometric radar imaging mechanism of oceanic surface currents," IEEE Trans. Geosci. Remote Sens., vol. 38, no. 1, pp. 446-458, Jan. 2000.

[10] S. Suchandt and H. Runge, "High-resolution surface current mapping using TanDEM-X ATI," in Proc. 10th Eur. Conf. Synth. Aperture Radar (EUSAR'14), Berlin, Germany, Jun. 3-5, 2014, pp. 1-4

[11] R. Romeiser and H. Runge, "Measuring the temporal autocorrelation function of backscattered X-band signals from the ocean with TanDEMX," in Proc. 10th Eur. Conf. Synth. Aperture Radar (EUSAR'14), Berlin, Germany, Jun. 3-5, 2014, pp. 1-3.

[12] H. Breit, M. Lachaise, U. Balss, C. Rossi, T. Fritz, and A. Niedermeier, "Bistatic and interferometric processing of TanDEM-X data," in Proc. 9th Eur. Conf. Synth. Aperture Radar (EUSAR'12), Nürnberg, Germany, Apr. 23-26, 2012, pp. 93-96.

[13] M. Costantini, "A novel phase unwrapping method based on network programming," IEEE Trans. Geosci. Remote Sens., vol. 36, no. 3, pp. 813-821, May 1998

[14] S. Bontemps et al. (2011, Feb.). GLOBCOVER 2009: Products Description and Validation Report. ESA, Université catholique de Louvain, Belgium [Online]. Available: http://due.esrin.esa.int/files/p68/ GLOBCOVER2009_Validation_Report_2.2.pdf

[15] D. P. Dee et al., "The ERA-interim reanalysis: Configuration and performance of the data assimilation system," Quart. J. Roy. Meteorol. Soc., vol. 137 , no. 656 , pp. 553-597, Apr. 2011.

[16] J. Lawrance, H. Kofoed-Hansen, and C. Chevalier, "High-resolution metocean modelling at EMEC's (UK) marine energy test sites," in Proc. EWTEC'09, Uppsala, Sweden, Sep. 7-10, 2009, 13 pp.

[17] H. Runge et al., "Mapping of tidal currents with SAR along-track interferometry," in Proc. IEEE Int. Geosci. Remote Sens. Symp. (IGARSS'04), Anchorage, AK, USA, Sep. 20-24, 2004, pp. 1156-1159.

[18] R. Romeiser, H. Runge, S. Suchandt, R. Kahle, C. Rossi, and P. S. Bell, Quality assessment of surface current fields from TerraSAR-X, and TanDEM-X along-track interferometry, and doppler centroid analysis," IEEE Trans. Geosci. Remote Sens., vol. 52, no. 5, pp. 2759-2771, May 2014.

[19] Fundy Ocean Research Center for Energy (FORCE), Halifax, Nova Scotia, Canada [Online]. Available: http://fundyforce.ca, accessed on February 27, 2015

[20] Fisheries and Oceans Canada (DFO), Ottawa, ON, Canada [Online]: Available: http://tides.gc.ca, accessed on February 27, 2015.

[21] S. Rahmstorf, "Thermohaline ocean circulation," in Encyclopedia of Quaternary Sciences, S. A. Elias, Ed. Amsterdam, The Netherlands: Elsevier, 2006.
[22] G. Feng, S. Jin, and J. M. Sanchez Reales, "Antarctic circumpolar current from satellite gravimetric models ITG-GRACE2010, GOCE-TIM3 and satellite altimetry," J. Geodyn., vol. 72, pp. 72-80, 2013.

[23] Helmholtz Alliance Remote Sensing and Earth System Dynamics, Helmholtz Association, Berlin, Germany [Online]: Available: http://hgfeda.de, accessed on February 27, 2015.

[24] W. A. Qazi, W. J. Emery, and B. Fox-Kemper, "Computing ocean surface currents over the coastal california current system using 30-minute lag sequential SAR images," IEEE Trans. Geosci. Remote Sens., vol. 52, no. 12, pp. 7559-7580, Jun. 2014.

[25] M. Gade, B. Seppke, and L. Dreschler-Fischer, "Mesoscale surface current fields in the Baltic Sea derived from multi-sensor satellite data," Int. J. Remote Sens., vol. 33, no. 10, pp. 3122-3146, May 2012.

[26] S. J. Frasier and A. J. Camps, "Dual-beam interferometry for ocean surface current vector mapping," IEEE Trans. Geosci. Remote Sens., vol. 39, no. 2, pp. 401-414, Feb. 2001.

[27] N. Otsu, "A threshold selection method from gray-level histograms," IEEE Trans. Syst. Man Cybern., vol. 9, no. 1, pp. 62-66, Jan. 1979.

[28] J. Schulz-Stellenfleth, I. Hajnsek, and S. Lehner, "Use of TanDEM-X in a squinted split antenna mode configuration to retrieve 2-D current, and ocean wave information," in Proc. IEEE Int. Conf. Geosci. Remote Sens. Symp. (IGARSS'06), Denver, CO, USA, Jul. 31/Aug. 4, 2006, pp. 24082411.

[29] P. Lopez-Dekker et al., "Experimental bidirectional SAR ATI acquisitions of the ocean surface with TanDEM-X," in Proc. 10th Eur. Conf. Synth. Aperture Radar (EUSAR'14), Berlin, Germany, Jun. 3-5, 2014, pp. 1-4.

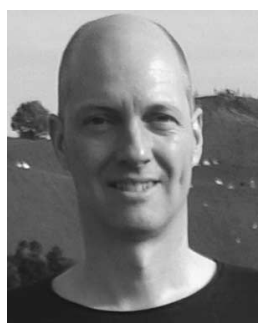

Steffen Suchandt received the Dipl.-Ing. degree in electrical engineering from the University of Rostock, Rostock, Germany, in 1995.

From 1996 to 1998, he worked in the field of biomedical engineering. Since 1998, he has been with the Remote Sensing Technology Institute, German Aerospace Center (DLR), Oberpfaffenhofen, Germany, working on numerous aspects of interferometric synthetic aperture radar (SAR) processing. $\mathrm{He}$ was engaged in data calibration, processor development, and digital elevation model processing of the Shuttle Radar Topography Mission (SRTM) and worked on the design and development of an automatic traffic information extraction system for TerraSAR-X data. He has a wide experience in SAR interferometry, ground moving-target indication, signal processing, and software development. Furthermore, he is currently responsible for the design of a SAR geodesy processing system. His research interests include ocean surface observations using multichannel spaceborne SAR.

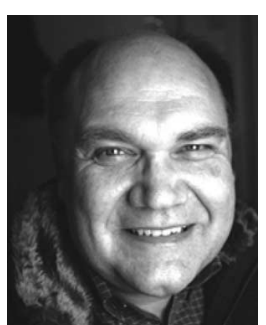

Hartmut Runge received the Dipl.-Ing. degree in communications technology from the University of Siegen, Siegen, Germany, in 1980.

He is a DLR Staff Member and has been working with the first digital SAR processor and on various aspects of SAR processing and applications. He was one of the inventors of the basic chirp scaling algorithm and the responsible system engineer of the data processing chain for the X-SAR missions and the Shuttle Radar Topography Mission (SRTM). In that mission, he was also the Principal Investigator for the along-track interferometry experiments and used this technique to reveil the potential for ocean current measurements. His research interests include geodetic SAR applications. 